\title{
Parcours figuratif de la femme dans la bande dessinée Aya de Yopougon de Marguerite Abouet et Clément Oubrerie
}

\author{
Goufo Zemmo \\ Université de Liège
}

\section{Introduction}

Depuis sa création dans les années $1825^{1}$, la bande dessinée (BD) s'est imposée comme un médium proposant des éléments de compréhension de la société (Dacheux 9-27) Domaine artistique manifestement surinvesti dans ses débuts par des productions au masculin, c'est avec peine que les auteures féminines y ont trouvé une place pour leurs créations. Cette masculinité en bande dessinée s'est trouvée encore plus criarde en Afrique subsaharienne. Il a fallu y attendre les années 2000, pour assister à la publication de la première bande dessinée féminine ${ }^{2}$. Cette dernière a contribué, de manière indirecte, à activer l'implication des femmes africaines dans le secteur du graphisme. De plus en plus, on assiste à une croissance timide des productions dites "féminines », notamment à travers l'éclosion de l'écriture et du dessin féminins. Ces productions féminines prennent davantage en charge la problématique de la femme africaine et abordent la question de la responsabilité énonciative de cette dernière dans le récit. En effet, il est remarquable de découvrir dans les différentes productions graphiques féminines que le récit est toujours narré et même porté par une héroïne $^{3}$, personnage principal. De ce fait, les auteures de bandes dessinées féminines poseraient implicitement la question de l'identité des sujets féminins dans l'espace social de leur représentation; l'espace de la société africaine dans un premier temps, celui de la bande dessinée dans un second temps. Aussi, pouvons-nous, nous interroger sur ce qu'il y aurait à découvrir dans ces récits de femme, ainsi que les processus textuels ou graphiques que mettent en place ces auteures pour optimiser l'accomplissement de la représentation des sujets féminins dans leurs bandes dessinées. Pourrait-on, au vu de tous ces processus, conclure à l'idée d'une bande dessinée féminine africaine engagée? Quelles valeurs pourraient véhiculer celle-ci?

Nous entendons traiter cette problématique dans la bande dessinée ivoirienne Aya de Yopougon. Ce qui nous intéresse ici, ce n'est pas de statuer

\footnotetext{
${ }^{1}$ Cette année est celle de la naissance des tous premiers dessins illustrés du genevois $\mathrm{R}$. Töpffer. Il est considéré à ce jour comme le Père de la BD. Cependant la date de parution des premiers albums de BD diffère selon le pays. Pour le cas de la France, ils apparaissent dès 1839 (M. Lajaunisse de Cham) et, pour le cas de l'Allemagne, dès 1860 avec les aventures de Max und Morit:

${ }^{2}$ Il s'agit de la BD de Fifi Mukuna intitulé Kisi le Collier

${ }^{3}$ Nous constatons cela dans La vie D'ébène Duta avec de Elyon's dont l'héroïne est Ébène, Aya de Yopougon de Marguerite Abouet dont l'Héroïne est Aya.
} 
sur la manière dont cette bande dessinée contribue à réfléchir sur les genres, soit en en donnant des caractéristiques déterminantes, soit en relevant les outils de compréhension de la société africaine, mais de partir des structures narratives de son récit pour comprendre comment s'y construit l'ascension identitaire de la femme africaine à travers l'architecture énonciative des sujets féminins.

\section{1. «Aya de Yopougon » : la bande dessinée}

\section{a. Le schéma sémio narratif d'" Aya de Yopougon "}

Aya de Yopougon est une bande dessinée d'origine ivoirienne. Elle est scénarisée par l'Ivoirienne, M. Abouet et dessinée par le français C. Oubrerie, époux de M. Abouet. Cette dernière naît effectivement en Côte d'Ivoire en 1971. Ses parents vont l'envoyer dès son adolescence (elle a douze ans) poursuivre ses études à Paris. Néanmoins, elle a déjà assimilé un certain nombre de connaissances de l'Afrique. Elle garde donc jalousement ses souvenirs de « Yopougon », son quartier d'enfance à Abidjan. À partir de 2005, commence la retransmission de ces souvenirs dans le roman graphique qui porte le titre Aya de Yopongon. Nous pourrions d'ailleurs le qualifier de $\mathrm{BD}$ autobiographique, dans ma mesure où, son récit comporte, à bien des égards, des similitudes avec sa biographie personnelle ${ }^{4}$. Elle y relate la vie d'un groupe d'adolescents ivoiriens, mais son souci premier est d'abord celui de «donner à voir une représentation plus réaliste de la Côte d'Ivoire que celle, misérabiliste, véhiculée par les médias européens » (Ajayi 2009).

Paru aux éditions Gallimard entre 2005 et 2010 en six tomes, Aya de Yopougon présente Aya, l'héroïne, comme étant une jeune fille africaine déterminée à ne pas suivre la série $C$, (Couture, Coiffure, chasse mari, etc.,) comme toutes les jeunes filles de son quartier. Elle réussit, avec beaucoup de peine à convaincre ses parents de lui donner la possibilité de s'inscrire en faculté de médecine pour échapper au mariage forcé auquel ces derniers la prédestinent. Loin d'être un abri sûr, l'université devient rapidement pour elle un cauchemar, puisqu'elle y est victime de harcèlement sexuel. L'enjeu devient celui de surmonter cette épreuve pour montrer à ses congénères qu'une femme africaine n'est pas seulement vouée aux rôles insignifiants, mais peut assurément être une tête pensante, agissante et même un leader dans la société.

La bande dessinée Aya de Yopougon se construit dans une perspective chronologique continue. Le déroulement de l'histoire est synchronique, c'est-à-dire constitué d'événements survenus à la même époque, quoique dans un espace social variant d'une séquence à une autre. La dynamique temporelle reste donc évolutive; ce qui pourrait au fil des tomes perdre certains lecteurs. On remarque donc en parcourant la bande dessinée que

\footnotetext{
${ }^{4}$ Ceci n'est qu'une hypothèse qui pourrait faire l'objet d'une étude indépendante concernant le caractère autobiographique ou fictif d' Aya de Yopongon.
} 
pour pallier à des incompréhensions ou à des manquements liés à l'édition sous forme de tomes, l'auteure fait recours régulièrement à des analepses pour restituer au lecteur des éléments qu'il aurait négligés ou oubliés au fil des parutions.

Au-delà de cette scission très cohérente du récit d'Aya de Yopongon, le lecteur peut rapidement se rendre compte qu'il s'y tisse un jeu sur l'altérité culturelle et les valeurs de la femme noire en général. Alliant subtilement au graphisme et à la richesse des thèmes évoqués, les couleurs chaudes des dessins (bruns, jaune, orangé, rouge, etc.), M. Abouet et C. Oubrerie permettent au lecteur de rentrer pleinement dans l'univers de l'Afrique dès le premier tome. Ils lui permettent également de s'interroger au-delà de tout, sur la condition existentielle des jeunes filles et femmes dont la présence et les rôles sont assez représentatifs dans cette bande dessinée.

\section{b. Une bande dessinée féministe?}

Comme nous l'avons souligné dans notre propos précédent, Aya de Yopougon n'est pas la première bande dessinée africaine créée par un auteur féminin. Cependant, contrairement à la bande dessinée Kisi le collier (2002), de Fifi Mukuna qui est pionnière en Afrique subsaharienne, son succès a contribué de manière significative à ouvrir un pan de voile sur l'univers de création des bandes dessinées féminines d'Afrique noire francophone. Il a également normalisé, en quelque sorte, la publication de bandes dessinées artistiques. Depuis lors, on en découvre de plus en plus, entre autres, La vie d'ébène Duta (tome1 et $2^{5}$ ) de Joëlle Ebongué dit Elyon's, Chronique de Brazzaville de Jussie Nsana... et certaines maisons d'éditions internationales telles que L'Harmattan, Gallimard, Glénat..., consacrent de plus en plus un espace privilégié à la diffusion des créations graphiques africaines. Pourtant, cet élan seul ne justifie pas le choix que nous avons porté sur ce roman graphique pour comprendre le militantisme d'auteures de bandes dessinées féminines africaines.

En effet, hormis le succès éditorial et même cinématographique ${ }^{6}$ qui la caractérise, la découverte sur les planches d'une multitude de sujets féminins pourrait être perçue comme un élément catalyseur qui force l'attention de celui qui plonge pour la première fois dans cet univers imagé. Bien sûr, il faut convenir que les personnages masculins y sont également représentés. Cependant, ils n'ont pas toujours le «bon rôle », et ce dernier, de manière générale, reste un rôle secondaire. En témoigne le rôle actantiel incarné par Moussa, dont la seule présence au début du récit consiste à frimer en voiture pour attirer les jeunes filles, ou encore Ignace qui mène une double vie conjugale à l'insu de son épouse, sans compter Koffi qui décide de prendre

\footnotetext{
${ }^{5}$ Le tome 2 est paru officiellement en Novembre 2015

${ }^{6}$ La bande dessinée Aya de Yopougon a également connu un très grand succès avec son adaptation en film d'animation en 2013. Il a reçu le César du meilleur film d'animation au festival d'Annecy la même année.
} 
pour seconde épouse Rita, une jeune fille d'une vingtaine d'années sa cadette. L'unique personnage masculin qui prend en charge un rôle principal est Monsieur Sissoko, le directeur général de la Solibra?. Du fait de son allure impressionnante et de sa fonction sociale, il s'impose le respect ${ }^{8}$ de son entourage. Son récit ou son histoire n'est cependant pas directement mêlé à celui de l'héroïne Aya et de ses amies (Bintou et Adjoua), mais correspond à ce que G. Genette (Genette 184) appellerait un récit enchâssé, c'est-à-dire un récit secondaire, distinct du récit principal, mais avec qui il partage des éléments communs. En plus de la relégation absolue des personnages masculins à des rôles secondaires, nous remarquons que, parmi les six tomes que comptent cette bande dessinée, quatre, dont les trois premiers et le sixième, présentent sur leur première de couverture des personnages féminins dont Aya accompagnée de Bintou et Adjoua, puis Rita et Félicité. Le quatrième tome, quant à lui, prend en charge le personnage masculin Innocent, représenté au milieu de deux femmes. Innocent est un coiffeur homosexuel spécialisé dans les coiffures féminines; de fait, son rôle actantiel, ainsi que ses traits physionomiques qui prêtent à confusion (visage fin, cheveux longs, sourcils épilés, etc.,) entraînent à première vue des questionnements sur la nature même de son genre (masculin ou féminin?). Le cinquième album présente, une fois de plus, Monsieur Sissoko en avant-plan, suivi au second plan de son épouse Simone et d'un autre personnage masculin. Il se déploie dans la bande dessinée, et ceci au fil des tomes, une omniprésence remarquable des personnages féminins. Ils agissent comme des ombres, se profilant entre les planches, toujours complices de cette pseudo-présence graphique. Pourtant, interrogée en 2013 sur une possible volonté féministe entre son héroïne (Aya) et elle, M. Abouet répond : « Je ne suis pas féministe. $\mathrm{Ou}$ du moins je suis féministe... quand cela m'arrange. Quand, pour les femmes, la liberté et la justice sont menacées, oui. Mais cela s'arrête là. » (De Rochebrune Interwiev, 2013). Cette assertion a le mérite de donner clairement le point de vue de M. Abouet sur la question d'un quelconque féminisme dont elle ne se revendique point. Mais doit-on présager dans la surreprésentation féminine de cette bande dessinée un engagement ou une volonté de lutter contre une quelconque injustice dont seraient victimes les femmes dans la société africaine?

Il s'agit donc de réinvestir le parcours figuratif de ces femmes dans Aya de Yopongon pour comprendre le processus par lequel, M. Abouet inscrit, à sa manière, sa bande dessinée dans le réaménagement de la substance représentationnelle de la femme dans la société africaine. Le personnage

\footnotetext{
${ }^{7}$ Il s'agit d'une grande société brassicole qui fabrique de la bière, très prisée par les jeunes de Yopougon.

${ }^{8} \mathrm{Il}$ représente mieux le tyran que le leader.

${ }^{9}$ Dans ce cas spécifique, le père d'Aya, Ignace, travaille pour Monsieur Sissoko. Ignace souhaiterait d'ailleurs qu'elle épouse Moussa le fils de son patron.
} 
d'« Aya » à qui est confiée la délégation absolue de tous les points de vue ${ }^{10}$ (elle est la narratrice principale) et dont le patronyme même, se trouve être le titre de la bande dessinée, donnerait à réfléchir sur la place qu'occupent les femmes dans ce récit de femme, ainsi que les traits figuratifs qui rendent compte de leur ascension identitaire dans ce roman graphique.

\section{Le parcours figuratif des femmes dans Aya de Yopougon}

Il se pose dès l'entame d'Aya de Yopougon, une construction architecturale de l'image de la femme africaine à travers le parcours figuratif des sujets féminins convoqués.

Défini par A. J. Greimas et J. Courtés comme « un enchaînement isotope corrélatif à un thème donné » (Dictionnaire 146), le parcours figuratif est double, à la fois liberté et contrainte pour l'instance énonciative dans la mesure où, lorsque la première figure est posée, elle n'en appelle que certaines, plus importantes, tout en excluant d'autres, tel que nous le verrons dans la suite de notre argumentaire. En effet, la surreprésentation des femmes, qu'il nous a été donné de voir plus haut, place ces dernières au cœur même de l'univers axiologique de cette bande dessinée. En parcourant la bande dessinée, on constate l'existence d'une cohésion textuelle qui scande le texte en trois énoncés bien distincts. Ces trois énoncés pourraient permettre de décrire le parcours figuratif des sujets féminins en tant qu'ascension, évolution dynamique d'un processus qui est tissé par l'auteure dès le premier tome jusqu'au dernier. Il s'agit de trois moments importants correspondant à la situation initiale, au déroulement et à la situation finale du récit. Nous les avons formulés comme suit : la vie des femmes à Yopongon au début de lintrigue, le récit du concours de "miss Yopougon » et le récit du barcèlement sexuel d'Aya. Ces trois moments rendent compte à notre avis des transformations identitaires des sujets féminins et correspondent aux trois parcours figuratifs qui les caractérisent c'est-à-dire, le fondement de l'altérité, la découverte d'un «moi » différent, et enfin la construction d'une nouvelle identité porteuse de signification.

\section{a. Le fondement de l'altérité : "la vie des femmes à Yopougon dans ses débuts"}

La séquence narrative que nous appelons «la vie des femmes à Yopougon dans ses débuts" pose la problématique de l'altérité en tant propriété négative au même titre que Barthes qui la qualifiait comme «le concept le plus antipathique au bon sens » (Barthes 45). Perçu sous différents aspects comme le rapport qui unit un élément $(A)$ à d'autres éléments $(A, B$, $\mathrm{C}, \mathrm{D} \ldots)$, le dispositif d'altérité se fonde sur le caractère de ce qui est autre.

\footnotetext{
${ }^{10}$ Le point de vue tel que nous l'entendons ici, correspond à la focalisation de G. Genette. Il s'agit de la procédure qui consiste à inscrire (ou à cerner) un acteur ou simplement une séquence dans des coordonnées spatio-temporelles très précises. L'acteur devient un sujet cognitif qui sait tout ou presque de ce qui se passe dans son environnement.
} 
Dans le cas de cette bande dessinée, le rapport d'altérité se crée dès le départ, à travers la relation qui lie les sujets féminins entre elles premièrement, et celle qui les unit, deuxièmement, aux sujets masculins.

La situation initiale du récit pose une image des filles adolescentes dont les connotations se résument à la séduction et aux réjouissances quotidiennes. Elles se rendent à des bals tous les soirs, et se donnent pour objectifs de séduire le plus grand nombre de jeunes hommes du village. C'est principalement le cas de Bintou et Adjoua qui n'hésitent pas à déjouer la vigilance des parents le soir pour aller « s'enjailler » (Faire la fête) ${ }^{11}$ dans les maquis (Restaurant pas cher où on peut manger et danser). Le récit pose ces jeunes filles dans un contexte social où l'éducation n'est pas une priorité. Sur la planche 4 du premier album (Tome 1, p. 4), on y découvre Adjoua prétendre à son frère aîné, qu'elle va traiter des exercices scolaires chez son amie; un subterfuge qui est découvert dans la suite du récit, dans laquelle elle est dévoilée dans un bal du quartier avec ses amis Adjona et Moussa. Ces deux jeunes filles incarnent véritablement l'insouciance et la naïveté de la jeunesse. Les sorties sont pour elles un impératif et l'auteure s'en sert pour présenter concrètement le rapport qui les unit à leur alter ego masculin. Il n'y est pas seulement question de divertissement, mais d'un besoin de se poser auprès des sujets masculins; entité par laquelle la société les aurait contraintes à se définir. Le rapport d'altérité, qu'elle pose, se fonde sur un personnage masculin en tant qu'être supérieur, capable de les conjoindre à une reconnaissance sociale; cette reconnaissance, à l'entame de la bande dessinée, passant inéluctablement par le mariage; unique moyen pour devenir une femme respectable et respectée dans l'espace des logiques sociales d'Abidjan.

Cette idée se profile notamment dans les trois premiers tomes de l'album et tout spécialement dans le premier tome. Dans celui-là, Aya s'offusquant devant l'attitude insouciante de ses amies, et les interpellant sur l'importance pour toutes jeunes filles de s'instruire, reçoit contre toute attente des réponses narquoises de Bintou et Adjoua. Bintou, la première lui répond:

«Eh Aya! Tu me fais rire dêh (exclamation ivoirienne.)! En tout cas moi j’aime la série $\mathrm{C}^{12}$. Je me vois déjà dans mon grand salon de coiffure acheté par mon mari...»,

Et Adjoua d'ajouter «Et moi donc, dans mon magasin de couture où toutes les plus grandes femmes d'Abidjan viendront se faire coudre leurs habits...) (Abouet ; Oubrerie, Aya I 18)

\footnotetext{
11 La majorité des mots étrangers présents dans cet article proviennent des idiolectes ivoiriens, du fait même de l'origine de cette bande dessinée. Cependant, grâce au phénomène sociolinguistique, beaucoup sont de plus en plus réappropriés par les citoyens d'autres pays africains.

${ }^{12} \mathrm{La}$ « série $\mathrm{C}$ » est une appellation ivoirienne pour qualifier ceux qui choisissent de faire de la Couture, de la Coiffure et de la Chasse plutôt que de poursuivre leur étude. Il faut dire que dans la société africaine, la couture et la coiffure sont dévalorisées par les intellectuels, puisqu'elles ne nécessitent pas toujours une formation académique.
} 
Force est de constater qu'à côté de cette indolence des femmes dans Aya de Yopongon, se dissimule toujours une présence masculine qui pourrait être considérée comme "défaitiste». Dans la suite du dialogue en effet, l'argumentaire d'Aya est stoppé par l'arrivée de Mamadou, le jeune homme charmeur du quartier dont la seule présence suffit à émouvoir toutes les filles. Alors qu'elle s'apprête à exposer son projet d'avenir qui consiste à devenir médecin, "mais moi aussi, je me vois plus tard... », ces propos sont coupés court par l'introduction de cette présence masculine qui inhibe si l'on peut dire toutes les capacités des jeunes filles à se découvrir elles-mêmes en tant qu'entité autonome et indépendante.

Fort de cela, émergent deux figures des sujets féminins : celle de la femme non-scolarisation et celle de la femme dépendante, dont le seul rapport d'altérité est étroitement affilié à la présence masculine. Ces deux figures sont renchéries par les représentations des femmes adultes qui, du fait de leur statut social monotone, contribuent davantage à la construction d'une référence péjorative de la femme dont les isotopies confirment les deux figures précédentes.

La femme que nous définissons ici comme adulte se trouve être, soit une épouse soit une mère d'un âge avancé. Ce rôle est incarné par les mamans de Bintou (Alphonsine), Aya (Fanta), et Adjoua (Korotoumou) auxquelles s'ajoutent Madame Simone Sissoko et Jeanne la maîtresse d'Ignace le père de Aya. Le parcours de Simone, Fanta, Korotoumou et Alphonsine est assez représentatif du statut de la femme traditionnelle. Elles sont toutes les quatre des femmes au foyer dont les seules tâches se résument à faire le ménage, la vaisselle, la cuisine et s'occuper des enfants. Seule Fanta cumule à ses rôles domestiques, celui de guérisseuse traditionnelle ${ }^{13}$ et de secrétaire de direction. Quoique Simone, Korotoumou et Alphonsine n'aient pas été scolarisées, le respect social leur est dû dans le quartier du fait de leur statut d'épouses. Contrairement à elles, Jeanne, secrétaire de direction, tout comme Fanta, ne reçoit aucune reconnaissance sociale, car elle est considérée comme mère célibataire.

En joignant à la majorité des sujets féminins les figures de la nonscolarisation ou de la sous-scolarisation, et d'une dépendance accrue aux sujets masculins, l'énoncé construit une figure de cette dernière dont l'extériorisation se résumerait à son rapport d'altérité, de dépendance à l'homme, comme nous le disions plus haut. Ce dernier lui donnerait alors sens et existence. Dans ce cas, leur relation n'est pas égalitaire puisque la figure féminine, telle qu'elle émerge à ce stade, se constitue comme inférieure. Elle se caractérise par ce que nous pourrions qualifier d'absence de personnalité.

\footnotetext{
${ }^{13}$ Il s'agit d'une personne qui n'a pas nécessairement fait des études de médecine, mais qui grâce à des expériences personnelles cumulées est capable de poser un diagnostic ou encore de prescrire un traitement efficace au moyen de plantes médicinales.
} 
Pourtant, cette première figure de la femme noire, telle qu'elle est offerte par le récit précurseur, n'est pas fortuite. En effet, c'est dans l'énonciation de ses isotopies que se posera le fondement de son ascension. Cette position qui l'infériorise n'étant en fait, qu'une base discursive pour sa construction future. Il s'agira pour la bande dessinée d'y prendre appui par la suite, pour poser la question du «moi » autonome de la femme dans Aya de Yopougon.

\section{b. La découverte d'un moi féminin: "le récit de "miss Yopougon" "}

La trame narrative que nous nommons «l'organisation de miss Yopougon» se pose comme un récit révélateur. Il s'institue comme une métaphore de la prise de conscience du statut précaire qui caractérise les femmes dans cet univers social. En effet, jusqu'ici Aya, dont la personnalité est très différente de celle de ses amies, se trouve toujours incomprise par celles-ci. Ce refus d'écouter que ses amies opposent, n'arrête pas pour autant la détermination d'Aya de les conscientiser sur les valeurs intrinsèques de la femme. Celles-ci résideraient notamment dans l'éducation et dans leur autonomie en tant que personnes. L'enjeu du récit s'inscrit dans la construction d'une position textuelle dans laquelle, Aya pourra se déployer, faisant de fait passer ses dires du statut de paroles à ceux de discours en acte. C'est ce dernier qui aide à la construction d'un sujet féminin en termes de reconnaissance, d'appropriation cognitive, et de pratique de soi.

Dans le troisième tome notamment, on assiste à l'organisation d'un concours de beauté (Miss Yopougon) dans le quartier. Bintou, Adjona et Félicité veulent y participer. Leur objectif est tout d'abord celui de séduire les hommes par la beauté de leur corps, et s'arracher, comme elles le disent, les «meilleurs prétendants» de Yopougon. Pourtant, ces jeunes filles, qui ne prennent pas leurs études au sérieux, seront rapidement désillusionnées par les organisateurs. Ces derniers privilégient d'abord l'intelligence des candidates en reléguant au second plan la beauté. Le concours signale l'isotopie de la beauté comme éphémère et préconise d'autres valeurs sociétales parmi lesquelles, l'éducation et l'entrepreneuriat au féminin. Il se pose pour ces jeunes filles la question de la vérité des valeurs et comme le précise J. Geninasca, dans un processus de reconnaissance, l'affirmation de l'identité apparaît comme le moteur de l'histoire, la seule en mesure de conférer un statut signifiant aux transformations, insensibles ou brutales, qui changent les rapports de l'homme et du monde. (46). "Miss Yopougon» situe la réussite des sujets féminins dans la réception et l'ancrage de ces nouvelles valeurs. La quête de la couronne devient le moteur qui conditionnera le faire discursif des jeunes filles.

Il devient primordial pour les sujets féminins de se doter des compétences nécessaires pour obtenir le succès au concours. C’est dans ce cadre que s'organisent autour d'Aya, des cours de soutien pour apporter une 
aide à Bintou, Adjoua et Félicité, dont les connaissances intellectuelles sont très moyennes. Ceci est un extrait d'une séquence de répétitions entre Aya et Bintou :

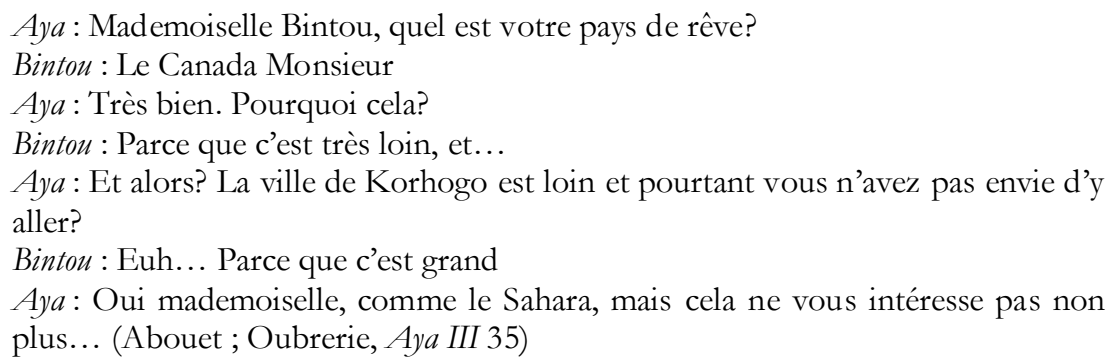

Dans cet extrait, nous remarquons que, même si le niveau des réponses ne satisfait pas encore aux exigences de l'entraîneuse $A y a$, ce qui ressort de cet échange reste la détermination de Bintou à se forger sa propre identité, et à nourrir des valeurs qui ne lui seront point enlevées. Même si au terme du concours aucune des trois jeunes filles ne remporte la couronne, cela ne constitue en rien un échec pour elles. La suite du récit met en place une nouvelle figure de la femme noire qui commence à se dévoiler par ses entreprises. On découvre des femmes qui s'affirment, l'une comme représentante d'une grande marque de cosmétique (Félicitê), l'autre (Adjoua) comme propriétaire d'un "maquis ", et Bintou, égale à elle-même, devient « conseillère en relation sentimentale ».

La découverte de l'existence de nouvelles compétences dans leur être amène ces jeunes femmes à se détacher peu à peu de la fixation qu'elle avait au départ des hommes, ceci pour mieux s'affirmer. C'est le cas de Adjoua qui, devenue propriétaire de son maquis, refuse de s'installer avec Mamadou le Père de son enfant. Devant l'insistance de ce dernier, elle répond avec autorité : «Mamadou, j'aime ma place : un maquis, un fils, de l'argent. Que demander de plus? » (Abouet ; Oubrerie, Aya IV 63)

Il commence à s'instituer à ce stade du récit, un dispositif d'identité de la jeune fille à travers l'acquisition de ces nouvelles compétences qui seront le moteur décisif de la construction identitaire dont elles font preuve à la fin de la bande dessinée.

\section{c. La construction d'une identité : "le harcèlement sexuel d'Aya "}

L'identité est étroitement liée à la notion de différence. C'est le caractère de ce qui individualise une personne, une communauté... Elle est étroitement rattachée au contexte qui donne à l'instance de mieux se définir. Comme nous l'avons montré en amont, le récit met au fil de son déroulement narratif une évolution des figures de la femme. Tout d'abord révélée comme sous-scolarisée et dépendante des hommes, elle trouve, par le biais du concours de «miss Yopougon », de nouvelles valeurs qui l'amène à penser et envisager sa propre identité. Toutefois, le fait que les sujets 
féminins aient conscience d'exister pour eux-mêmes, n'est pas le gage de l'acquisition de cette identité. Son authentification s'extériorise à travers la difficulté à laquelle fait, cette fois-ci, face Aya dans la suite du récit.

Considéré depuis le départ comme sujet narrateur qui sait tout, Aya incarne au mieux les valeurs identitaires auxquelles ses amies sont en quête. Pourtant, le harcèlement sexuel dont elle fait l'objet de la part de son professeur de biologie l'amène à une remise en question. Dans un processus d'identification en effet, la remise en question est posée comme l'essence même de l'émergence d'un « moi » authentique (Geninasca 48). Elle permet de mieux se construire et de mieux se positionner face à l'autre. Ainsi, deux issues sont proposées par le texte :

- Soit, elle cède aux avances du professeur et obtient son diplôme de médecine sans aucun effort, tout en perdant ces valeurs pour lesquelles elle lutte au quotidien.

- Soit, elle garde la tête haute et renonce à son rêve de devenir médecin.

Le bouleversement intérieur qui l'habite l'amène à se confier à ses amies Bintou et Adjoua. Ces dernières se transforment tout de suite en détectives pour lui trouver une nouvelle voie de sortie à cette situation. En positionnant, Aya en difficulté contre un personnage masculin, le récit donne la possibilité à Adjoua et Bintou de vérifier la compétence qu'elles ont acquise pendant et après le concours de beauté. Elles vont élaborer un plan dans le but de confondre le professeur. Ce dernier n'étant pas à son premier forfait, elles réussissent à retrouver une précédente victime qui accepte de servir d'appât. Des microphones lui sont posés sur le corps, et sa conversation enregistrée avec le professeur est transmise aux autorités policières. La supercherie du professeur est ainsi dévoilée au grand plaisir de toute la communauté universitaire féminine.

L'introduction du professeur de biologie comme opposant des sujets féminins dans le récit n'est pas accidentelle. Elle permet de confirmer cette nouvelle identité qu'elles ont acquise et de dévoiler une autre figure de la femme. Il est intellectuel et, à ce titre, son échec face à Adjoua et Bintou, confirme la revalorisation identitaire des femmes dans cette bande dessinée. À travers cette épreuve, émerge une femme réfléchie, rusée, et capable de rivaliser, non plus avec des hommes ordinaires, mais avec des intellectuels, auprès de qui elle n'aurait plus aucune frustration. Son rapport à l'homme dans cette dernière séquence n'est plus celui de sujet dominé, mais grâce à l'émergence de cette nouvelle identité, elle se positionne comme son semblable, son égal...

Quoique M. Abouet ne se revendique point féministe, l'ascension dont font preuve les sujets féminins dans Aya de Yopougon, montre une détermination à vouloir lutter contre des images stéréotypées auxquelles feraient face les femmes en Afrique, et dont le premier parcours aurait 
énuméré quelques-unes. En effet, le récit se déroule en 1977, c'est-à-dire quelques années après les indépendances en Afrique subsaharienne. Durant cette époque, et même après, la femme africaine est restée cloîtrée dans une sphère sociale qui ne lui concédait aucun droit. Victimes de disparités sociales telles que la relégation absolue au rôle de " domestique », l'absence au droit de vote, à l'éducation,... elle a eu de la peine à s'émanciper et à retrouver les mêmes droits que ses correspondants. Quoique les textes juridiques africains lui confèrent aujourd'hui, un statut égal à celui des hommes, la paupérisation sociale à son endroit demeure encore stable et imperturbable.

Le dessinateur franco-camerounais C. Ngalle Édimo l'affirmait lors d'une interview au festival d'Angoulême portant sur le rôle de la femme dans les bandes dessinées africaines :

Les femmes africaines ont encore des combats à mener pour s'émanciper, $[\ldots]$ dans la représentation de certains africains, la femme est inféodée à l'homme. Je pense que c'est une catastrophe. [...] Les choses bougent en Afrique peut-être même de façon un peu souterraine. Certains hommes vont se réveiller un jour et s'apercevoir que leur représentation est fausse, trop négative. (Tabary)

Dans une Afrique où, « les filles sont beaucoup plus opprimées, ont beaucoup plus de mal à s'en sortir », comme l'affirme M. Abouet (De Rochebrune) cette ascension discursive pourrait être une volonté d'amener les lectrices qui vivent des situations similaires à celles de ces jeunes filles à s'interroger sur l'investissement ou l'orientation à laquelle elles sont appelées à conjoindre.

Certaines lectrices ont d'ailleurs avoué que la réception favorable qu'elle avait eue de cette bande dessinée, avait été orientée en grande partie par les points de convergences entre les jeunes femmes représentées dans la bande dessinée et elles-mêmes. Ce dialogisme identitaire entre des personnages fictifs et ceux en chair et en os, montre bien que la bande dessinée de M. Abouet Aya de Yopongon a dépassé, le simple divertissement pour s'instituer comme une véritable littérature engagée qui traite de la problématique de la femme dans l'espace social africain.

\section{Conclusion}

L'objet de cette analyse était de comprendre l'ascension de la femme africaine à travers le parcours figuratif des sujets féminins dans la bande dessinée Aya de Yopongon de M. Abouet et C. Oubrerie. Si la surreprésentation des personnages féminins n'a pas été perçue comme une volonté féministe de son auteure, elle s'est, dans les analyses, concrétisée comme une volonté de cette dernière de lutter contre les injustices dont seraient victimes les femmes dans la société africaine. Le parcours des personnages féminins a permis la génération d'une figure féminine autonome et remplie de confiance. Cette autonomie est postulée dès le départ par Aya. Elle reflète dès son entrée en scène l'image d'une femme 
africaine moderne à laquelle rien n'échappe. Son intelligence et son sens de l'écoute la rendent tout de suite attachante. Ses traits graphiques sont à la fois fins, doux, et on y perçoit toute l'attention du dessinateur. Son parcours et celui de ses amies deviennent des «gestes indicateurs » qui pointent vers quelque chose de saisissable : l'identité de la femme, à la fois force dans le caractère, et douceur dans le trait graphique. 


\section{Bibliographie}

Abouet, Marguerite. Oubrerie, Clément. Aya de Yopongon 1. Gallimard, 2005. Abouet, Marguerite. Oubrerie, Clément. Aya de Yopongon 2. Gallimard, 2006. Abouet, Marguerite. Oubrerie, Clément. Aya de Yopongon 3. Gallimard, 2007. Abouet, Marguerite. Oubrerie, Clément. Aya de Yopongon 4. Gallimard, 2008. Abouet, Marguerite. Oubrerie, Clément. Aya de Yopongon 5. Gallimard, 2009. Abouet, Marguerite. Oubrerie, Clément. Aya de Yopougon 6. Gallimard, 2010. Ajayi, Angela. "World - Africa- Interview - Drawing on the Universal in Africa: An Interview with Marguerite Abouet". 9 mars 2009. Wild Rever Review, bttp://wmw.wildriverreview.com/interview/drawing-universalafrica/marguerite-abouet/ ajayi-angela. Consulté le 31 sept. 2015.

Barthes, Roland. Mythologies, Seuil Points/Essais, 1970.

Benveniste, Émile. Problèmes de linguistique générale I. Gallimard, 1966.

Cassiau-Haurie Christophe. Meunier, Christophe. Cinquante années de bandes dessinées en Afrique francophone. L'Harmattan, 2010.

Dacheux, Éric. "Présentation générale. La bande dessinée. Une représentation critique de notre monde de représentation." Ed. Éric Dacheux. Bande dessinée et Lien social, CNRS ÉDITIONS, 2014, pp. 927

De Rochebrune, Renaud. "Interview avec Marguerite Abouet", 17 Juillet 2013, http:// www.jeuneafrique.com/136786/culture/ marguerite-abouet-lesivoiriennes-sont-moins-libres-qu-avant/. Consulté le 2 déc. 2015.

Genette, Gérard. Figures III. Seuil, 1972.

Greimas, Algirdas Julien et Joseph Courtés. Sémiotique. Dictionnaire raisonné de la théorie du langage. vol. 1, Hachette, 1979.

Geninasca Jacques. La parole littéraire. PUF, 1997.

Goufo Zemmo. «L'énonciation spatiale de la femme dans la BD africaine », Journée des jeunes chercheurs, 23 sept. 2015, Liège.

Goufo Zemmo., "Représentation des genres dans les BD féminines africaines contemporaines ", Congrès international de sémiotique visuelle, 10. sept. 2015, Université de Liège, Liège.

Tabary, Constance. (Le rôle de la femme dans la BD africaine, selon C. Édimo) Festival de la BD d'Angoulême, 6 fév. 2011, bttp:/ / www.toukimontreal.com/2011/02/06/le-role-de-la-femme-dans-labede-africaine-selon-christophe-edimo/. Consulté le 2 déc. 2015. 